J Am Med Dir Assoc. 2016 January 1; 17(1): 85-88. doi:10.1016/j.jamda.2015.10.017.

\title{
Infection Prevention and Control Programs in United States Nursing Homes: Results of a National Survey
}

\author{
Carolyn T. A. Herzig, PhDa , Patricia W. Stone, PhDa , Nicholas Castle, PhD ${ }^{b}$, Monika \\ Pogorzelska-Maziarz, $\mathbf{P h D}^{\mathrm{c}}$, Elaine L. Larson, $\mathbf{P h D}^{\mathrm{a}}$, and Andrew W. Dick, $\mathbf{P h D}^{\mathrm{d}}$ \\ aColumbia University School of Nursing, 630 West $168^{\text {th }}$ Street, Mail Code 6, New York, NY \\ 10032, USA \\ bDepartment of Health Policy and Management, University of Pittsburgh, A610 Crabtree Hall, \\ Pittsburgh, PA 15261, USA \\ cJefferson College of Nursing, Thomas Jefferson University, 130 S. Ninth Street, Room 847, \\ Philadelphia, PA 19107, USA \\ ${ }^{\mathrm{d} R A N D}$ Corporation, 20 Park Plaza, Suite 920, Boston, MA 02116, USA
}

\begin{abstract}
Objectives-The objectives of this study were to (1) obtain a national perspective of the current state of nursing home (NH) infection prevention and control (IPC) programs and (2) examine differences in IPC program characteristics for NHs that had and had not received an infection control deficiency citation.
\end{abstract}

Design-A national cross-sectional survey of randomly sampled NHs was conducted and responses were linked with Certification and Survey Provider Enhanced Reporting (CASPER) and NH Compare data.

Setting-Surveys were completed and returned by 990 NHs (response rate 39\%) between December 2013 and December 2014.

Participants-The person in charge of the IPC program at each NH completed the survey.

Measurements-The survey consisted of 34 items related to respondent demographics, IPC program staffing, stability of the workforce, resources and challenges, and resident care and

Corresponding author: Carolyn T. A. Herzig, Columbia University School of Nursing, 630 West $168^{\text {th }}$ Street, Mail Code 6 , New York, NY, 10032, Phone: (212) 342-3912, Fax: (212) 305-3659, cth2115@ cumc.columbia.edu. Alternate corresponding author: Patricia W. Stone, Columbia University School of Nursing, 630 West $168^{\text {th }}$ Street, Mail Code 6, New York, NY, 10032, Phone: (212) 305-1738, Fax: (212) 305-3659, ps2024@ cumc.columbia.edu.

Conflict of interest: MPM served as a paid consultant to Becton, Dickinson and Company. The consulting work was unrelated to the research presented in this manuscript. The remaining authors have no conflicts of interest to disclose.

Human subjects protection: All procedures were approved by the Columbia University Medical Center, University of Pittsburgh, and RAND Corporation Institutional Review Boards and a waiver of written informed consent was obtained.

Publisher's Disclaimer: This is a PDF file of an unedited manuscript that has been accepted for publication. As a service to our customers we are providing this early version of the manuscript. The manuscript will undergo copyediting, typesetting, and review of the resulting proof before it is published in its final citable form. Please note that during the production process errors may be discovered which could affect the content, and all legal disclaimers that apply to the journal pertain. 
employee processes. Facility characteristics and infection control deficiency citations were assessed using CASPER and NH Compare data.

Results-Most respondents had at least two responsibilities in addition to those related to infection control (54\%) and had no specific IPC training (61\%). While many practices and processes were consistent with infection prevention guidelines for $\mathrm{NHs}$, there was wide variation in programs across the US. About $36 \%$ of responding facilities had received an infection control deficiency citation. NHs that received citations had infection control professionals with less experience $(P=.01)$ and training $(P=.02)$ and were less likely to provide financial resources for continuing education in infection control $(P=.01)$.

Conclusion-The findings demonstrate that a lack of adequately trained infection prevention personnel is an important area for improvement. Furthermore, there is a need to identify specific evidence-based practices to reduce infection risk in NHs.

\section{Keywords}

Nursing homes; long-term care; infection prevention; healthcare-associated infections; infection prevention training

\section{INTRODUCTION}

Healthcare-associated infections (HAI) are common in nursing homes (NHs) and contribute significantly to resident morbidity and mortality; ${ }^{1,2}$ however, little is known regarding common practices related to HAI prevention in this setting. It is mandated that all skilled nursing facilities/NHs have an individualized infection prevention and control (IPC) program. ${ }^{3}$ The Centers for Medicare and Medicaid Services (CMS) addresses these expectations during annual inspection surveys conducted for certification purposes; currently about $38 \%$ of US NHs receive an infection control deficiency citation each year., ${ }^{4,5}$

Guidelines for infection prevention in NHs provide recommendations on processes related to IPC program structure and function, resident care, and employees. ${ }^{3,6-9}$ Previous researchers have found that most NHs lack professionals who are adequately trained ${ }^{10}$ and identified wide variation across NHs in personnel dedicated to infection prevention, the adoption of recommended activities, and the use of standardized infection surveillance definitions. ${ }^{5,10-12}$ However, to date, most studies of infection control practices and processes in US NHs have had small sample sizes and limited geographic representation. There is a critical need to better understand practices related to HAI prevention in NHs. In order to gain insight in this area, the objectives of this study were to (1) obtain a national perspective of the current state of IPC programs in NHs and (2) examine differences in IPC programs for NHs that had and had not received an infection control deficiency citation.

\section{METHODS}

A cross-sectional survey of randomly sampled US NHs was conducted as part of the Prevention of Nosocomial Infections and Cost Effectiveness in Nursing Homes (PNICENH; R01NR013687) study. Eligible NHs were non-specialized, free-standing facilities with between 30 and 900 beds identified in the Online Survey, Certification, and Reporting 
(OSCAR) data. Between December 2013 and December 2014, staggered mailings and a modified Dillman technique including an initial mailing of the survey with an invitation letter, reminder postcards, and a last chance communication were used for recruitment. The person in charge of the IPC program at each NH was invited to complete the survey. To increase participation, \$20 gift cards were sent with each survey and respondents had the opportunity to be entered into a raffle to win an Apple iPad.

The 34-item survey (available upon request) included primarily closed-ended questions and was developed by adapting and modifying a survey used to evaluate IPC programs in acute care settings, ${ }^{13}$ guidelines for infection control specific to $\mathrm{NHs},{ }^{3}$ and results of a qualitative study of IPC programs in NHs. ${ }^{14}$ Content validity was confirmed by our study team and advisory board that includes experts in the field, and infection control professionals in three NHs; the survey was pilot tested in three additional NHs. The survey included items related to respondent demographics and staffing of the IPC program, stability of the workforce, resources and challenges, and resident care and employee processes. Survey responses were linked with concurrent Certification and Survey Provider Enhanced Reporting (CASPER; formerly called OSCAR) data to evaluate facility characteristics and NH Compare data to evaluate staffing levels, infection control deficiency citations (F-tag 441), quality of care deficiency citations (F-tags 0309-0312, 0314, 0316-0319, 0321-0325, 0328-0330, 0333, and/or 0353), and quality measures.

Descriptive statistics were computed using $\chi^{2}$, Fisher's exact, $t$ or Wilcoxon-Mann-Whitney tests, as appropriate. Characteristics of NHs that responded to the survey were compared with those of non-respondents. Additionally, differences in IPC program characteristics for NHs that had and had not received an infection control deficiency citation were evaluated. A significance level of 0.05 was set a priori and all analyses were conducted using SAS version 9.3 (SAS Institute, Cary, NC). All research procedures were approved by our Institutional Review Boards.

\section{RESULTS}

The survey was sent to 2,514 NHs and 990 completed surveys were returned (39\% response rate). About $39 \%$ and $65 \%$ of sampled facilities had received infection control and quality of care citations, respectively. Responding and non-responding NHs did not differ based on number of beds, occupancy, metropolitan setting, or chain affiliation. However, responding NHs had lower levels of licensed practical nurse (LPN) staffing and percent Medicare residents, were more likely to be nonprofit and located in the northeast, and were less likely to have received infection control or quality of care citations. Respondents and nonrespondents also differed on several quality measures (Table A1).

\section{Nursing home infection prevention and control program staffing}

Respondent demographics, IPC program staffing, and stability of the workforce are presented in Table A2. Respondents in $84 \%$ of NHs were RNs and, on average, had 11 years of experience conducting infection control-related work in any $\mathrm{NH}$ and 5 years in their current facility; fewer years of experience in their current $\mathrm{NH}$ was associated with receiving an infection control citation $(P=.01)$. Most respondents $(54 \%)$ had at least two 
responsibilities in addition to infection control, most commonly nursing administration, staff education, and employee health. Most respondents (61\%) had no specific training in infection control and lack of training was associated with receiving a citation $(P=.02)$. Training included taking a state or local course (26\%), a national Association for Professionals in Infection Control and Epidemiology (APIC) course (9\%), and Certification in Infection Control (3\%).

On average, respondents reported spending 29\% of their time on infection control activities; the most time-consuming were infection surveillance, tracking antibiotic use, and staff education. NHs with infection control citations spent more time on vaccination and immunization $(P=.05)$ and less time on infection control policy development $(P=.006)$. Regarding turnover, $41 \%, 42 \%$, and $39 \%$ of $\mathrm{NHs}$ reported having at least three people fill the roles of infection control professional, Director of Nursing, and $\mathrm{NH}$ administrator, respectively, during the previous three years; NHs with citations had higher Director of Nursing and $\mathrm{NH}$ administrator turnover $(P=.03$ and .04 , respectively).

\section{Nursing home infection prevention and control program resources and challenges}

IPC program resources and challenges are listed in Table A3. Most NHs had an infection control committee that met regularly and most included a nurse administrator, $\mathrm{NH}$ administrator, and a medical director; facilities with infection control citations were less likely to have a staff physician as a committee member $(P=.04)$. One-third of NHs were involved in an infection prevention collaborative (i.e., group focused on reducing HAIs in NHs by implementing reduction strategies, tracking progress, and providing feedback). Facilities with citations were more likely to be in a collaborative focused on reducing methicillin-resistant Staphylococcus aureus $(P=.04)$ and less likely to be in one focused on reducing hospitalizations $(P=.04)$. Finally, urinary tract infections $(96 \%)$, pneumonia/upper respiratory tract infections (83\%), and skin and soft tissue infections (35\%) were ranked as the three greatest infection challenges.

\section{Nursing home infection prevention and control program processes}

Table A4 summarizes resident care and employee processes. Lists of residents with infections were most frequently maintained in a log-book and/or as a paper list (76\%); other methods included keeping an electronic spreadsheet or database (40\%) and using a graphical map $(25 \%)$. There was variation in the types of information used to determine when a resident has an infection. Most NHs used clinical or laboratory cultures and physician/nurse practitioner diagnosis (69\%); many also used the updated McGeer criteria (41\%), Centers for Disease Control and Prevention (CDC) National Healthcare Safety Network (31\%), and/or Minimum Data Set (24\%) definitions. NHs with infection control citations were less likely to use the CDC definitions $(P=.007)$. Respondents were most frequently notified about potentially infected residents through the daily report (81\%); new antibiotic prescription reports were also a common method of notification (49\%) and their use was associated with receiving a citation $(P=.01)$. Most NHs monitored environmental cleaning practices and hand hygiene compliance via direct observation. In $77 \%$ of NHs in which hand hygiene compliance was monitored, feedback on compliance was provided to staff. 
While most NHs provided staff training on infection control topics at new employee orientation or when an issue arose ( $75 \%$ and $72 \%$. respectively), regularly scheduled trainings and updates were less common; NHs with infection control citations were less likely to schedule trainings on a weekly/biweekly/monthly basis $(P=.03)$. Almost all provided face-to-face trainings and/or in-services and other methods included posting flyers on care units (78\%) and/or computer- or video-based training tools (49\%), the latter of which was associated with receiving a citation $(P=.04)$. About half of $\mathrm{NHs}$ provided financial resources for continuing education opportunities in infection control. Opportunities included attendance at a meeting or conference (32\%) or external trainings $(25 \%)$. Not providing financial resources was associated with receiving a citation $(P=.01)$ and NHs with citations were less likely to have used resources for external trainings $(P=.04)$.

\section{DISCUSSION}

Here we present a national perspective of the state of IPC programs in US NHs. Overall, infection control professionals reported spending about 12 hours per week on infection control activities. Other studies have reported substantial variation in personnel resources devoted to infection control ${ }^{5,11}$ and further research is needed to determine required staffing when accounting for facility size and resident acuity. This is particularly relevant given the recently proposed regulations by $\mathrm{CMS}^{15}$ to require that facilities designate an Infection Prevention and Control Officer (IPCO) for whom the IPC program is their main responsibility. ${ }^{15}$ Furthermore, training in infection control is lacking. Low levels of training have been reported in Maryland long-term care facilities ${ }^{10,12}$ and a qualitative study showed that infection control professionals typically had minimal formal training but were viewed as experts. ${ }^{14}$ Finally, NHs in states in which the Department of Health provided access to training resources were less likely to receive infection control deficiency citations. ${ }^{4}$ These findings demonstrate training is an area requiring policy guideline development.

Urinary tract infection, pneumonia/upper respiratory tract infection, and skin and soft tissue infection were ranked as the three greatest infection challenges and are also the most common infections in NHs. ${ }^{1,2}$ Methods and processes used to collect and maintain information about infections, identify infected residents, notify staff about infected residents, and provide training and updates to staff varied widely and only half of facilities provided staff with financial resources for infection control education opportunities. It is important to determine what processes are effective in reducing HAI rates.

The results demonstrate that infection control professional experience and training, as well as staff training and continuing education, are important in terms of meeting regulatory requirements. However, most processes and practices did not vary based on whether a NH received an infection control citation. This was surprising because infection control is consistently among the top ten most common types of deficiencies ${ }^{16,17}$ and NHs might address this through changes in practice. It is possible that the survey did not capture requirements that, if not met, would result in an F-tag 441. It is also possible that NHs do not change practice in order to avoid receiving infection control citations if they are not enforced at a high level of severity. ${ }^{16,18}$ Importantly, it is not known whether meeting regulatory requirements for infection control programs results in reduced infection rates and 
additional research is needed to evaluate which IPC program characteristics impact overall resident care.

This study has several limitations. Although the sample size exceeded or was similar to other surveys of IPC programs in NHs, ${ }^{5,10-12}$ the response rate was moderate $(39 \%)$ so the results might not be generalizable. Respondents differed from non-respondents in certain characteristics and, in particular, respondents were less likely to have received infection control and quality of care citations indicating the sample consisted of better than average performing facilities. Nonetheless, variations in processes and practices were identified. The survey data were self-reported and although it was requested that the person in charge of the IPC program complete the survey, it is possible that respondents were not knowledgeable about the program. Because data were cross-sectional it was not possible to establish temporality when evaluating associations between IPC program characteristics and receiving an infection control citation. Finally, to evaluate those associations multiple comparisons were made and some of the observed significant associations might have happened by chance.

\section{CONCLUSION}

Although we found that many practices and processes were consistent with guidelines there was wide variation in NH IPC programs across the US. These findings suggest that the development of specific and evidence-based guidelines is needed. Additionally, infection control professionals had multiple responsibilities and lacked specific training and these are important areas for improvement. Future studies are needed to ascertain predictors of high infection rates in NHs in order to identify best practices related to infection prevention.

\section{Supplementary Material}

Refer to Web version on PubMed Central for supplementary material.

\section{Acknowledgments}

The authors would like to express their gratitude to the research team (John Engberg, Jasmine Travers, and Ragnhildur Bjarnadottir) and our advisory board (Lona Mody, Steven Schweon, Philip Smith, and Nimalie Stone) for their contributions to this study.

Funding source: This study was funded by the National Institute of Nursing Research (R01NR013687). The content is solely the responsibility of the authors and does not necessarily represent the official views of the National Institute of Nursing Research or the National Institutes of Health. The study sponsor had no role in the design or conduct or the study; in the collection, analysis, or interpretation of the data; in the writing of the manuscript; or in the decision to submit the manuscript for publication.

\section{References}

1. Dwyer LL, Harris-Kojetin LD, Valverde RH, et al. Infections in long-term care populations in the United States. J Am Geriatr Soc. Mar; 2013 61(3):342-349. [PubMed: 23496650]

2. Strausbaugh LJ, Joseph CL. The burden of infection in long-term care. Infect Control Hosp Epidemiol. Oct; 2000 21(10):674-679. [PubMed: 11083186]

3. Smith PW, Bennett G, Bradley S, et al. SHEA/APIC guideline: infection prevention and control in the long-term care facility, July 2008. Infect Control Hosp Epidemiol. Sep; 2008 29(9):785-814. [PubMed: 18767983] 
4. Cohen CC, Engberg J, Herzig CT, et al. Nursing Homes in States with Infection Control Training or Infection Reporting Have Reduced Infection Control Deficiency Citations. Infect Control Hosp Epidemiol. Sep $9.2015: 1-2$.

5. Ye Z, Mukamel DB, Huang SS, et al. Healthcare-associated pathogens and nursing home policies and practices: results from a national survey. Infect Control Hosp Epidemiol. Jul; 2015 36(7):759766. [PubMed: 25797334]

6. American Medical Directors Association. Common Infections in the Long-Term Care Setting Clinical Practice Guideline. Columbia, MD: AMDA; 2011.

7. Nicolle LE. Infection control in long-term care facilities. Clin Infect Dis. Sep; 2000 31(3):752-756. [PubMed: 11017825]

8. Richards CL Jr. Preventing antimicrobial-resistant bacterial infections among older adults in longterm care facilities. J Am Med Dir Assoc. Mar-Apr;2005 6(2):144-151. [PubMed: 15871891]

9. Richards CL Jr. Infection control in long-term care facilities. J Am Med Dir Assoc. Mar; 2007 8(3 Suppl):S18-25. [PubMed: 17336871]

10. Roup BJ, Roche JC, Pass M. Infection control program disparities between acute and long-term care facilities in Maryland. Am J Infect Control. Apr; 2006 34(3):122-127. [PubMed: 16630974]

11. Mody L, Langa KM, Saint S, Bradley SF. Preventing infections in nursing homes: a survey of infection control practices in southeast Michigan. Am J Infect Control. Oct; 2005 33(8):489-492. [PubMed: 16216667]

12. Roup BJ, Scaletta JM. How Maryland increased infection prevention and control activity in longterm care facilities, 2003-2008. Am J Infect Control. May; 2011 39(4):292-295. [PubMed: 21458109]

13. Stone PW, Pogorzelska-Maziarz M, Herzig CT, et al. State of infection prevention in US hospitals enrolled in the National Health and Safety Network. Am J Infect Control. Feb; 2014 42(2):94-99. [PubMed: 24485365]

14. Stone PW, Herzig CT, Pogorzelska-Maziarz M, et al. Understanding infection prevention and control in nursing homes: A qualitative study. Geriatr Nurs. Jul-Aug;2015 36(4):267-272. [PubMed: 25794923]

15. Centers for Medicare \& Medicaid Services. [Accessed August 13, 2015] Reform of Requirements for Long-Term Care Facilities: Proposed Rules (CMS-3260-P). 2015. http://www.gpo.gov/ fdsys/pkg/FR-2015-07-16/pdf/2015-17207.pdf

16. Office of Inspector General. [Accessed September 8, 2015] Nursing Home Deficiency Trends and Survey and Certification Process Consistency (OEI-02-01-00600). 2003. http://oig.hhs.gov/oei/ reports/oei-02-01-00600.pdf

17. Office of Inspector General. [Accessed September 8, 2015] Trends in Nursing Home Deficiencies and Complaints (OEI-02-08-00140). 2008. http://oig.hhs.gov/oei/reports/oei-02-08-00140.pdf

18. Castle NG, Wagner LM, Ferguson-Rome JC, et al. Nursing home deficiency citations for infection control. Am J Infect Control. May; 2011 39(4):263-269. [PubMed: 21531271] 